\title{
Treatment Switch Patterns and Healthcare Costs in Biologic-Naive Patients with Psoriatic Arthritis
}

Jashin J. Wu • Corey Pelletier · Brian Ung • Marc Tian •

Ibrahim Khilfeh · Jeffrey R. Curtis

Received: November 26, 2019 / Published online: March 5, 2020

(C) The Author(s) 2020

\section{ABSTRACT}

Introduction: We compared treatment switch patterns and healthcare costs among biologicnaive patients with psoriatic arthritis (PsA) who initiated apremilast or biologics.

Methods: A 1:2 propensity score match was used to adjust administrative claims data for adults initiating apremilast or biologics from January 1, 2014, to September 30, 2016, for possible selection bias. Patients had at least 12 months of pre- and post-index continuous enrollment in the Optum Clinformatics ${ }^{\mathrm{TM}}$ Data Mart database. Outcomes included switch frequency, days to switch, adherence on index treatment, and healthcare costs (total and per patient per month). Switch rate was defined as the proportion of patients who switched to a new treatment after initiation of the index

Enhanced Digital Features To view enhanced digital features for this article go to https://doi.org/10.6084/ m9.figshare.11830302.

\section{J. J. Wu}

Dermatology Research and Education Foundation, Irvine, CA, USA

C. Pelletier $(\bowtie) \cdot$ B. Ung · M. Tian · I. Khilfeh

Celgene Corporation, 86 Morris Avenue, Summit, NJ 07901, USA

e-mail: cpelletier@celgene.com

J. R. Curtis

University of Alabama at Birmingham, 1720 2nd

Avenue S, Birmingham, AL 35294, USA treatment, and days to switch was calculated as the days between initiation of the index treatment and initiation of the new treatment. Adherence was calculated using the proportion of days covered and the medication possession ratio. The $t$ test and chi-square, Kaplan-Meier, and Wilcoxon rank-sum tests were used to evaluate differences between the cohorts.

Results: Patient characteristics and switch rates were similar between the matched apremilast $(n=170)$ and biologic $(n=327)$ cohorts. After matching, patient characteristics were similar between the matched cohorts. The 12-month switch rates were similar for patients initiating apremilast versus those on biologics $(17.7 \%$ vs. $25.1 \%, P=0.06)$. This trend was similar at 6 months $(7.7 \%$ vs. $13.2 \%, P=0.07)$ and 18 months $(24.4 \%$ vs. $29.3 \%, P=0.33)$. Regardless of treatment switching, 12-month total healthcare costs were lower with apremilast versus biologics (all: $\$ 28,423$ vs. $\$ 41,178, P<0.0001$; switched: $\$ 39,803$ vs. $\$ 51,517, P=0.0040$; did not switch: $\$ 25,984$ vs. $\$ 37,717, P<0.0001$ ).

Conclusions: Biologic-naive patients with PsA who initiated apremilast had switch rates similar to biologic users and significantly lower healthcare costs, regardless of treatment switching. 


\section{PLAIN LANGUAGE SUMMARY}

Psoriatic arthritis (PsA) is a chronic inflammatory disease that affects an estimated $30 \%$ of psoriasis patients who use systemic therapy. Symptoms of PsA, such as joint swelling and tenderness, can be painful and disabling and may worsen quality of life. PsA can also impart a substantial economic burden. Treatment for moderate to severe PsA often involves the use of systemic oral medications (e.g., conventional systemic treatments such as methotrexate or targeted systemic treatments such as apremilast) or biologic therapy given by injection or infusion. Because PsA symptoms and responses to treatment can vary, patients may switch treatments over time. More research is needed to better understand how switching treatments affects healthcare costs among patients starting treatment with apremilast or a biologic for PsA. This study compared treatment switching and healthcare costs among patients with PsA who had never been treated with a biologic and who started treatment with apremilast or a biologic for PsA. Rates of treatment switching at 12 months were similar for patients starting treatment with apremilast versus those starting a biologic. Patients starting treatment with apremilast had significantly lower total healthcare costs compared with those starting a biologic, even if they later switched to a biologic. Healthcare costs calculated per patient per month (PPPM) were also lower with apremilast versus biologics, driven by lower PPPM pharmacy costs. These findings suggest that starting treatment with apremilast may be an effective and cost-effective strategy for managing PsA, even for patients who later switch to a biologic.

Keywords: Adherence; Apremilast; Biologics; Healthcare costs; Psoriatic arthritis; Rheumatology

\section{Key Summary Points}

Why carry out this study?

Patients with psoriatic arthritis who experience treatment failure may switch therapies during long-term treatment.

Limited real-world data are available regarding the effects of treatment patterns on healthcare costs among patients treated with apremilast.

This analysis used administrative health claims data from patients in the Optum Clinformatics ${ }^{\mathrm{TM}}$ Data Mart database to compare treatment switching and its effects on healthcare costs among biologic-naive patients with psoriatic arthritis who initiated treatment with apremilast or a biologic.

\section{What was learned from the study?z}

No difference was observed in switch rates among patients treated with apremilast or a biologic, with neither cohort reaching a greater than $50 \%$ switch rate $(P=0.2083)$, and mean 12-month total healthcare costs were significantly lower among those initiating apremilast versus biologics, regardless of treatment switching (all: $\$ 28,423$ vs. $\$ 41,178, P<0.0001$; switched: $\$ 39,803$ vs. $\$ 51,517, P=0.0040$; no switch: $\$ 25,984$ vs. $\$ 37,717, P<0.0001)$, with similar results observed in the 6- and 18-month post-index periods.

Mean per patient per month healthcare costs overall and by treatment patterns were lower for apremilast versus biologic cohorts in the 6-, 12-, and 18-month postindex periods, and the cost advantage with apremilast was driven by lower per patient per month outpatient pharmacy costs.

Starting treatment with apremilast may be a cost-effective strategy for managing psoriatic arthritis in biologic-naive patients, even in those who later switch to a biologic agent. 


\section{INTRODUCTION}

Psoriatic arthritis (PsA) is a chronic, systemic, and inflammatory disease that affects an estimated $30 \%$ of psoriasis patients who use systemic therapy and has an estimated prevalence of $0.3-1.0 \%$ in the general population [1]. Manifestations of PsA, including swollen and tender joints, pain, enthesitis and dactylitis, and skin involvement, can be disabling and often impair quality of life [2-5]. Patients with PsA also experience fatigue and work productivity loss [6], as well as common comorbidities such as cardiovascular disease, obesity, diabetes, depression, and anxiety $[4,6,7]$, which can contribute to both disease and economic burden [8]. Estimates of the direct annual healthcare costs related to PsA from a recent claimsbased analysis and systematic reviews have ranged widely from $\$ 3693$ to $\$ 26,883$ per patient; however, studies varied by year, country of study, payer type, severity of PsA, and costing methodology [9-11].

Patients with PsA have significant functional impairment with progressive joint damage; thus, treatment is generally focused on controlling disease activity to improve patient outcomes and prevent disease progression [12]. Initial treatment with non-steroidal anti-inflammatory drugs, with or without local corticosteroid injections, is recommended for patients with mild disease [12]. More active disease requires the use of conventional systemic disease-modifying anti-rheumatic drugs such as methotrexate, targeted synthetic disease-modifying anti-rheumatic drugs such as apremilast, or biologic agents that target tumor necrosis factor (TNF) alpha and interleukin (IL)12, IL-23, and IL-17A [5, 13-16]. Biologic agents have demonstrated efficacy in clinical trials and are often recommended for the treatment of patients with active PsA [12]; however, some may be associated with safety concerns, such as increased risk of infections [17-19]. Apremilast (Celgene Corporation, Summit, NJ, USA), an oral phosphodiesterase 4 inhibitor approved by the US Food and Drug Administration (FDA) in 2014, is indicated for the treatment of adult patients with moderate to severe plaque psoriasis who are candidates for phototherapy or systemic therapy and for adult patients with active PsA [20]. Through the inhibition of phosphodiesterase 4, apremilast subsequently partially inhibits the production of several cytokines implicated in the pathogenesis of psoriatic disease, including TNF $\alpha$, IL-23, and interferon- $\gamma$, unlike biologic agents that target a single inflammatory mediator [21-23]. Phase 3 clinical trials have demonstrated the efficacy and acceptable safety profile of apremilast in the treatment of patients with active PsA and showed clinically meaningful improvements in PsA disease severity and physical function $[13,24,25]$. An increased risk of serious infections or malignancies has not been reported with apremilast [13, 22, 24, 25], and treatment with apremilast does not require laboratory monitoring [26]. Diarrhea, headache, nausea, upper respiratory tract infection, nasopharyngitis, and vomiting were the most commonly reported adverse events in apremilast clinical trials $[13,24,25]$. On the basis of published pricing, apremilast is also less costly than biologic agents, including injectable biologics recently approved to treat active PsA (i.e., ixekizumab, secukinumab) [22, 27]. Furthermore, apremilast is an oral agent, and patients may prefer oral over injectable therapies [28]. The American College of Rheumatology guidelines recommend that initiating apremilast for the treatment of PsA should depend on the patient's disease history or preference for oral route of administration [17].

Because the severity of PsA varies over time and disease progression can result in irreversible joint damage $[1,5]$, patients who experience treatment failure may switch therapies during long-term management of their disease [29]. In a multinational survey of patients with psoriasis and PsA, most patients were not receiving treatment with any medication for their psoriasis or PsA (i.e., no systemic treatment or topical treatment) and many with psoriasis or PsA reported discontinuing systemic treatments because of concerns about safety and tolerability or loss of efficacy [28]. Many patients also reported cost as a reason for switching from biologic therapy. In the absence of head-to-head randomized controlled trials comparing the effectiveness of 
apremilast with specific biologic agents for the treatment of PsA, real-world studies can evaluate differences in outcomes, such as persistence, adherence, and healthcare costs, which are difficult to assess in randomized clinical trials. Considering that treatment switching may occur as a result of a lack of treatment response or tolerability concerns $[28,30]$ and that parameters such as dosing schedules, route of administration, and healthcare costs can affect or be associated with clinical outcomes, claims-based data on treatment patterns can augment our understanding of the overall effectiveness of treatments for PsA [31]. In recent claims-based analyses of data from biologic-naive patients initiating treatment with apremilast or a biologic for psoriasis, persistence rates for apremilast and biologics were similar and apremilast demonstrated a cost advantage (based on paid amounts of adjudicated claims) over biologics for patients who were persistent and for those who were not persistent (i.e., those who discontinued or switched from their index treatment) [32]. A similar study also found similar treatment persistence and switch rates and lower healthcare costs (including all adjudicated claims) among biologicnaive psoriasis patients receiving treatment with apremilast versus a biologic [33]. However, studies assessing treatment persistence or discontinuation rates may fail to adequately capture the true rate and costs associated with treatment switching. Additional research is needed to further determine the economic impact of switching treatments among users of apremilast and biologic therapies. The aim of the current study was to compare treatment switch patterns among biologic-naive patients with PsA who initiated treatment with apremilast versus a biologic. A second objective was to evaluate the total cost of care in these two cohorts overall and by treatment switch status.

\section{METHODS}

\section{Study Design and Data Source}

This retrospective, observational cohort study used US administrative health claims data from patients in the Optum Clinformatics ${ }^{\mathrm{TM}}$ Data
Mart (CDM) database (Optum Clinformatics Data Mart, Eden Prairie, MN, USA) for the United Health Group, a large national health insurance plan with both commercial and Medicare Advantage health plan data. Over 10 years (January 2007 to September 2017), the database represents a geographically diverse population spanning all 50 states and including 55 million unique lives covered. The CDM database provides detailed utilization, outcomes, and cost data for healthcare services provided in the inpatient, outpatient, and outpatient pharmacy settings and is statistically deidentified in accordance with the Health Insurance Portability and Accountability Act of 1996 as per 45 Code of Federal Regulations 164.506(d) (2)(ii)(B). Institutional review board approval was not required because this study used only de-identified patient records and did not involve the collection, use, or transmittal of individually identifiable data, as dictated by Title 45 Code of Federal Regulations 46.101(b)(4).

\section{Study Population}

The study population included biologic-naive patients who initiated a new treatment with apremilast or a biologic agent that was FDAapproved for PsA with or without a claim for psoriasis between January 1, 2014, and September 30, 2016. The date of treatment initiation was defined as the index date. The study consisted of a pre-index period (defined as the 12 months before the index date) and a postindex period (defined as a minimum of 12 months after the index date). Prior receipt of apremilast and biologic agents was identified in the baseline period and all prior data (if available) back to January 1, 2011; patients may have had prior treatment episodes with a biologic agent before this date. Patients were included if they were at least 18 years of age on the index date, had initiated a new treatment with apremilast or a biologic agent (adalimumab, certolizumab, etanercept, golimumab, infliximab, ixekizumab, secukinumab, and ustekinumab [other biologics were not used because they were not approved by the FDA at the time 
of the study]) for PsA between January 1, 2014, and September 30, 2016, had at least two medical claims with an International Classification of Diseases, Ninth or Tenth Revision, Clinical Modification (ICD-9-CM or ICD-10-CM) diagnosis of PsA (ICD-9-CM code 696.0/ICD-10-CM code L40.5) with or without a claim for psoriasis (ICD-9-CM code 696.1/ICD-10-CM code L40) during the 12 months before the index date, and had a minimum of 12 months of continuous enrollment before and after the index date. Patients were excluded from this analysis if they had a diagnosis of or were diagnosed with other biologic-indicated autoimmune conditions in the pre-index or post-index period (e.g., ulcerative colitis, Crohn's disease, rheumatoid arthritis or other inflammatory polyarthropathies [Felty's syndrome], ankylosing spondylitis, juvenile idiopathic arthritis), if they had a diagnosis of cancer at any time in the preindex or post-index period, or if they did not have at least a 12-month (if prior data were available) baseline period "clean" of any index agent.

\section{Study Outcomes}

Outcomes analyzed in this study were treatment patterns (switch frequency, days to switch, and adherence while on index treatment) and healthcare costs (total and per patient per month [PPPM]). A switch could be either a switch to a different treatment (apremilast or a biologic) or the addition of a new treatment for combination therapy. The switch rate was defined as the proportion of patients who switched to a new regimen at 3,6, $9,12,15,18,21$, and 24 months after the initiation of the index treatment. At 3, 6, 9, and 12 months, switch rates were calculated using the full patient sample; at 15, 18, 21, and 24 months, switch rates were calculated only within the subgroup of patients with at least 15 months, at least 18 months, at least 21 months, and at least 24 months of post-index follow-up, respectively, including full medical and pharmacy coverage. Days to switch was defined as the time from the date of initiation of the index treatment to the date of initiation of a new biologic treatment (or apremilast). Adherence while on index treatment was calculated using the proportion of days covered (PDC; number of days in the period "covered"/number of days in the period; variable denominator) and the medication possession ratio (MPR; [total days supply in period]/[last fill date - first fill date + last fill date days supply]).

Healthcare costs were calculated using Optum's standard pricing methodology for all claims data in the CDM database and included inpatient admissions, outpatient visits or services (including emergency department visits, office visits, and laboratory tests), and outpatient pharmacy claims. The standard pricing algorithm, applied to de-identified claims data, was used to estimate standard prices that reflect allowable payment amounts (i.e., the insurance payment amounts) across all provider services. Total healthcare costs at 6,12 , and 18 months were defined as the total sum of healthcare costs over a span of 6,12 , or 18 months since initiating treatment, and PPPM total healthcare costs were defined as the total average monthly healthcare costs while patients remained on the index treatment. Total healthcare costs and total PPPM healthcare costs were calculated on the basis of 6-, 12-, and 18-month post-index periods (within the total patient sample for the 6- and 12-month analyses and within the subgroup of patients with at least 18 months of post-index follow-up for the 18-month analysis). Healthcare costs were calculated and reported for the overall study population, as well as for patients who switched and did not switch therapies; among patients who switched, costs were calculated before and after the switch.

\section{Statistical Analysis}

Patients in the apremilast and biologic cohorts were propensity score matched up to $1: 2$ to adjust for possible selection bias using the nearest neighbor matching with a caliper width equal to 0.2 of the standard deviation of the logit of the propensity score [34]. A ratio of 1:2 was selected to increase statistical power and to 
maximize the number of apremilast and biologic users within the database. The propensity score was defined as the probability of being treated with apremilast given the baseline characteristics. Logistic regression was used to estimate the propensity score for individual patients with the following variables: age, gender, region, payer (commercial or Medicare Advantage), plan type, index year, prescriber specialty (dermatology, rheumatology, or other), Charlson comorbidity index score, preindex healthcare costs, number of prior systemic agents (i.e., acitretin, cyclosporine, methotrexate, sulfasalazine, and leflunomide), and prior use of non-steroidal anti-inflammatory drugs, cyclooxygenase- 2 inhibitors, corticosteroids, or phototherapy. These measures were identified as variables that were possibly related to both exposure cohort membership and outcome and were selected on the basis of the literature [32, 35] and available data from the CDM database.

Baseline patient demographics, clinical characteristics, and treatment patterns between the apremilast and biologic cohorts were compared using a $t$ test for continuous variables and chi-square test for categorical variables. Variables were summarized descriptively using means and standard deviations (SDs) for continuous variables and counts and percentages of patients for categorical variables; descriptive results were stratified by index agent and number of prior treatments. To account for patientcensoring (for analyses for more than 12 months), Kaplan-Meier methodology was utilized to test for differences in switch rates between the apremilast and biologic cohorts. Wilcoxon rank-sum tests were used to evaluate the cost differences for patients initiating apremilast compared with those initiating a biologic therapy. A $P$ value of 0.05 or less was considered statistically significant.

\section{RESULTS}

\section{Patients}

A total of 1576 patients from the CDM database were identified as having a new treatment episode with apremilast or a biologic for PsA between January 1, 2014, and September 30, 2016, and were eligible for study inclusion. After excluding patients who were biologic or apremilast experienced (42\%) and applying the remainder of the inclusion and exclusion criteria, the apremilast and biologic patients were then propensity score matched up to $1: 2$ to provide a final matched sample comprising 170 biologic-naive patients in the apremilast cohort and 327 patients in the biologic cohort (Fig. 1). Biologic index treatments were adalimumab $(n=166)$, etanercept $(n=83)$, ustekinumab $(n=37)$, infliximab $(n=16)$, certolizumab $(n=15)$, golimumab $(n=5)$, and secukinumab $(n=5)$. Prior to propensity score matching, the apremilast-treated patients were generally older (53.5 vs. 49.1 years of age, $P<0.0001$ ), were female $(60.3 \%$ vs. $49.1 \%, P<0.0067)$, and had a higher prevalence of certain comorbidities (i.e., cardiovascular disease $[P=0.0265]$, osteoporosis $[P=0.0147]$, hepatotoxicity $[P=0.0374]$, post-menopausal symptoms $[P=0.0016])$ than the biologic-treated patients (Table 1).

After matching, patient demographics and clinical characteristics, comorbidities (except for fibromyalgia), and history of prior therapies were similar in the two cohorts (Table 1). The mean age was 53.0 years (apremilast) and 52.3 years (biologics); 60.0\% (apremilast) and 56.3\% (biologics) of patients were female, and the mean Charlson comorbidity index scores were 1.4 (apremilast) and 1.3 (biologics). In both cohorts, the majority of patients were white and the most common comorbidities were hypertension, osteoarthritis, and cardiovascular disease.

\section{Treatment Patterns}

Among those who switched in the 12-month post-index period, the mean number of days to switch was similar between the apremilast and biologic cohorts (201 days vs. 181 days, $P=0.38$ ). Switch rates for patients initiating apremilast were similar at 6 months $(7.7 \%$ vs. $13.2 \%, P=0.07), 12$ months $(17.7 \%$ vs. $25.1 \%$, $P=0.06)$, and 18 months $(24.4 \%$ vs. $29.3 \%$, $P=0.33)$ compared with patients initiating biologics; at 9 months, significantly lower 


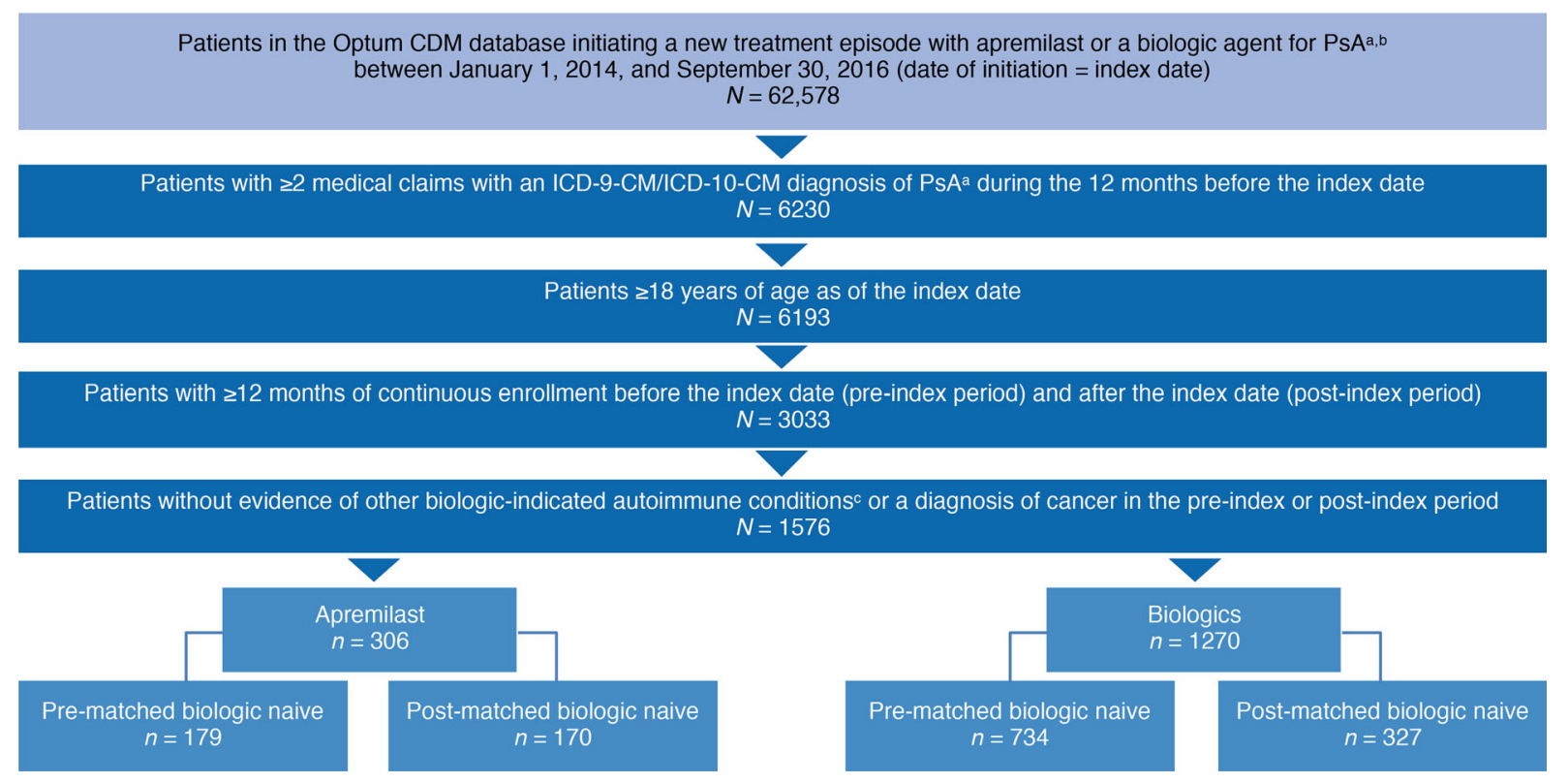

Fig. 1 Disposition of patients with PsA. ${ }^{a}$ With or without a claim for psoriasis. ${ }^{\mathrm{b}}$ Adalimumab, certolizumab, etanercept, golimumab, infliximab, ixekizumab, secukinumab, and ustekinumab. 'Ulcerative colitis, Crohn's disease, rheumatoid arthritis and other inflammatory polyarthropathies (Felty's syndrome), ankylosing spondylitis,

switch rates were observed in the apremilast versus biologic cohort $(12.9 \%$ vs. $20.2 \%$, $P=0.0448$ ) (Fig. 2).

While on index treatment, no significant difference in mean adherence rates was observed between the apremilast and biologic cohorts. The mean (SD) PDC while on index treatment was $0.84(0.17)$ for apremilast users versus $0.82(0.19)$ for biologic users $(P=0.39)$, and the mean (SD) MPR while on index treatment was $0.86(0.17)$ for apremilast users versus $0.84(0.20)$ for biologic users $(P=0.48)$.

No significant difference was observed in switch rates among all patients treated with apremilast or a biologic, as neither cohort reached a greater than 50\% switch rate $(P=0.21$; analysis based on all available postindex data) (Fig. 3).

\section{Overall and PPPM Costs}

At baseline, mean (SD) total healthcare costs per month in the matched apremilast and biologic

and juvenile idiopathic arthritis. $C D M$ Clinformatics ${ }^{\mathrm{TM}}$ Data Mart, ICD-9-CM International Classification of Diseases, Ninth Revision, Clinical Modification, ICD-10$C M$ International Classification of Diseases, Tenth Revision, Clinical Modification, $P S A$ psoriatic arthritis

cohorts were \$927 (\$983) and \$1000 (\$1263), respectively $(P=0.95$; Table 1$)$. Patients initiating apremilast had significantly lower mean 12-month total healthcare costs than those initiating biologics $(\$ 28,423$ vs. $\$ 41,178$, $P<0.0001$ ) (Fig. 4). This cost difference was largely attributed to differences in mean total outpatient pharmacy costs, which were significantly lower with apremilast than biologics at 12 months; differences between treatments in mean inpatient and total outpatient costs (including office visits, emergency department visits, laboratory testing, diagnostics, and infusions) during this time were not significant (Table 2). Patients initiating apremilast also had lower mean total healthcare costs overall than those initiating biologics during the 6-month post-index period $(\$ 15,226$ vs. $\$ 22,761$, $P<0.0001)$ and the 18 -month post-index period $(\$ 42,069$ vs. $\$ 57,188, P<0.0001)$ (Fig. 4$)$. At 6 months, mean total outpatient pharmacy and total outpatient costs were significantly lower for apremilast users than for biologic users, but differences in mean inpatient costs were not 


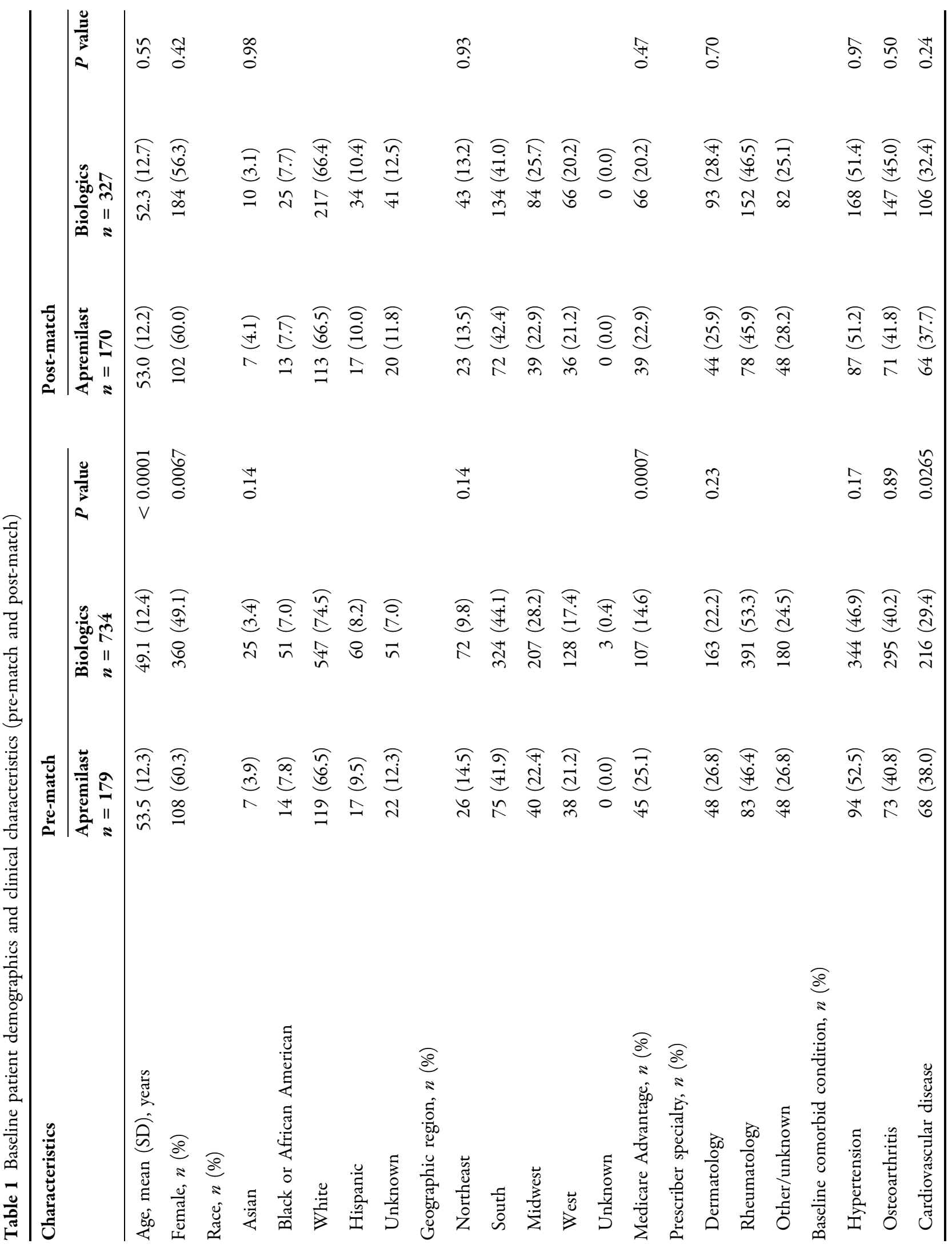




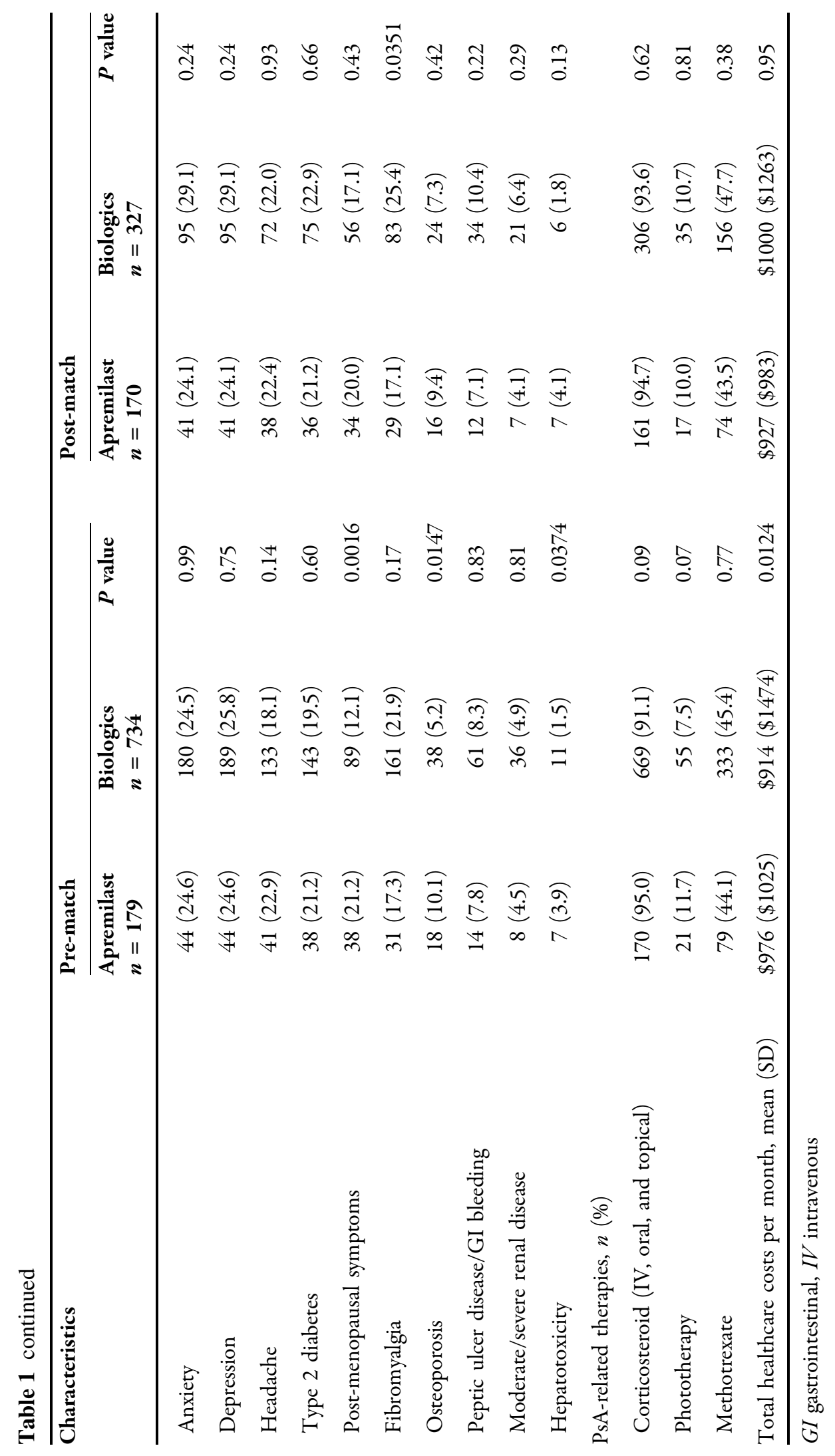




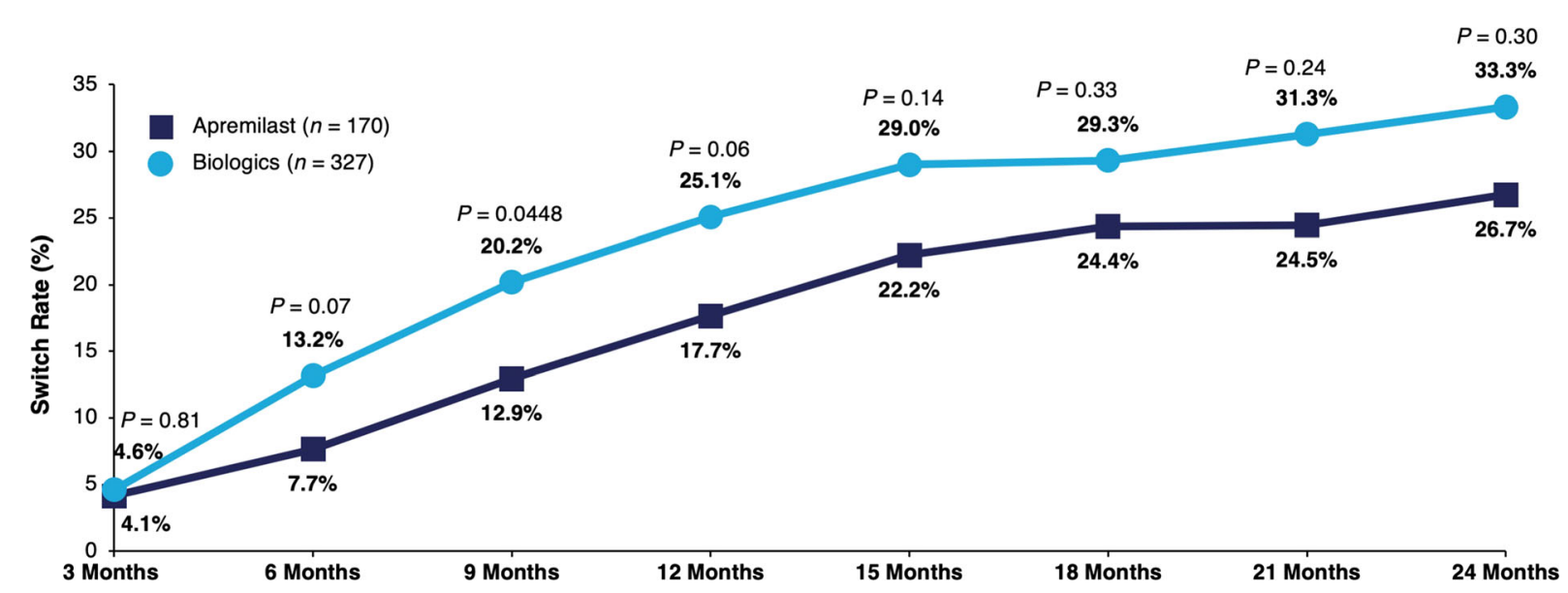

Fig. 2 Switch rates among biologic-naive patients with psoriatic arthritis initiating apremilast or biologics during the 0- to 24-month follow-up period. Switch rates were calculated within the subgroup of patients during the 0 - to 15-month post-index follow-up period (apremilast: $n=144$; biologics: $n=262$ ), during the 0 - to 18 -month

post-index follow-up period (apremilast: $n=119$; biologics: $n=208)$, during the 0 - to 21 -month post-index follow-up period (apremilast: $n=94$; biologics: $n=163$ ), and during the 0 - to 24-month post-index follow-up period (apremilast: $n=86$; biologics: $n=135$ )

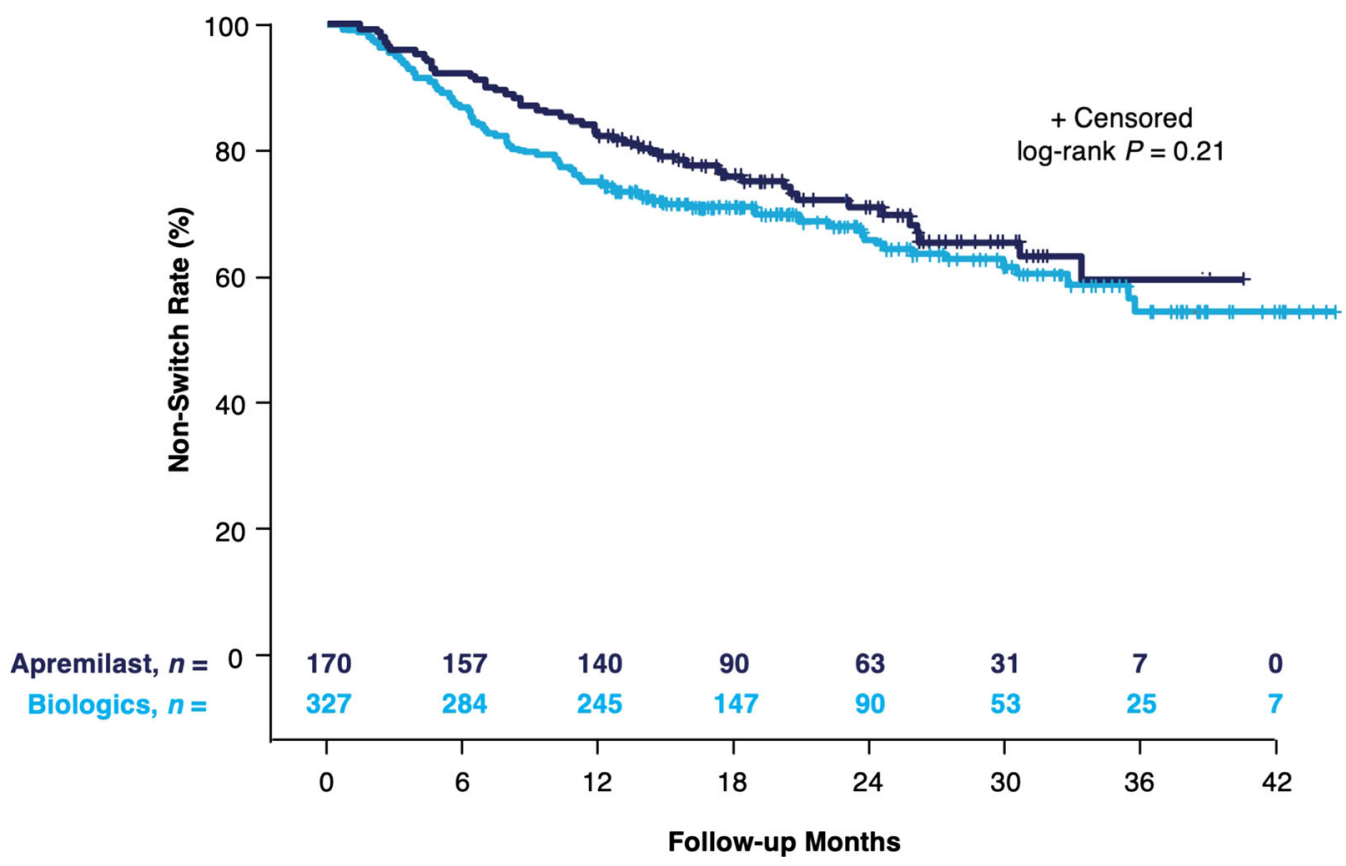

Fig. 3 Kaplan-Meier plot of treatment switch rates in biologic-naive patients with psoriatic arthritis, with number of patients at risk

significant. At 18 months, mean total outpatient pharmacy costs were significantly lower for apremilast users, but no significant differences in inpatient and total outpatient costs were seen (Table 2). Although some point estimates for mean inpatient cost outcomes were numerically lower for the biologic cohort than for the apremilast cohort, none of these were 


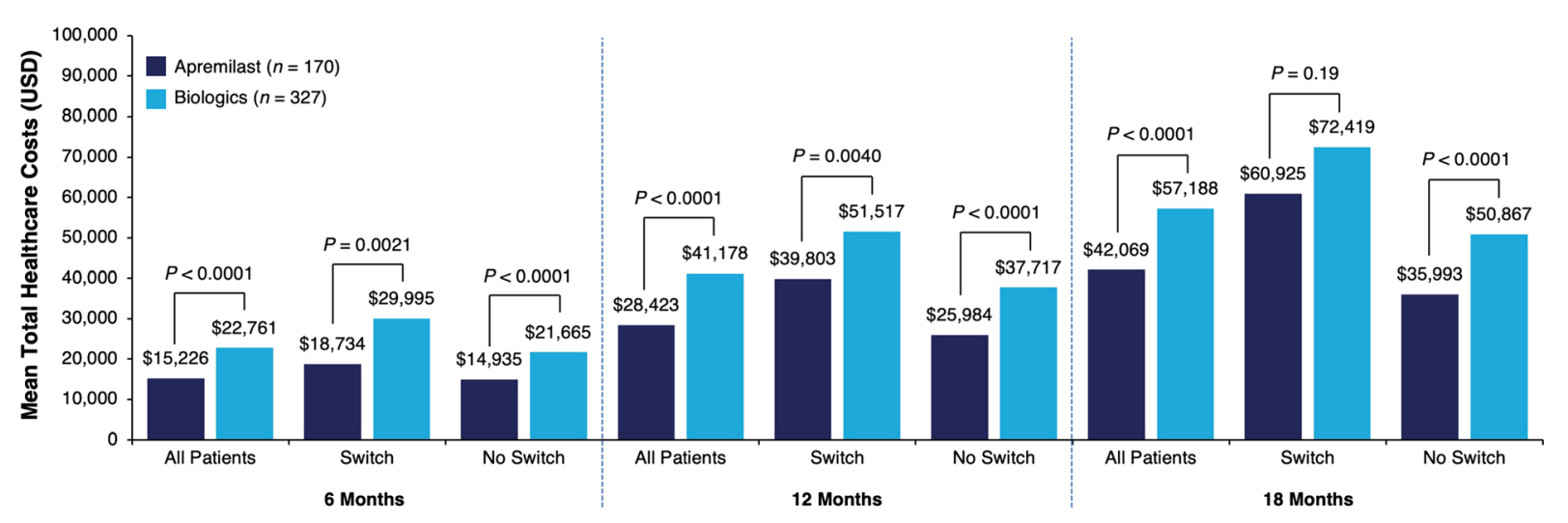

Fig. 4 Mean total healthcare costs ${ }^{\text {a }}$ over the 6-, 12-, and 18-month post-index periods, overall and by treatment pattern. aptum's standard pricing algorithms are applied to the de-identified claims data in the Optum

statistically significant (Table 2). Mean PPPM healthcare costs overall were significantly lower for the apremilast cohort versus the biologic cohort during the 12-month post-index period (\$2367 vs. \$3430, $P<0.0001 ;$ Table 2). In each cohort, total and PPPM healthcare costs were highest at 6 months and lowest at 18 months.

\section{Costs Among Patients Who Switched Treatments}

Significantly lower mean total healthcare costs were observed with apremilast versus biologics regardless of whether patients switched $(\$ 39,803$ vs. $\$ 51,517, P=0.0040)$ or did not switch $(\$ 25,984$ vs. $\$ 37,717, P<0.0001)$ treatments during the 12-month post-index period (Fig. 4). Mean total outpatient pharmacy costs were significantly lower with apremilast than with biologics among patients who did not switch treatments. However, these costs were similar among apremilast and biologic patients who did switch treatments. Mean inpatient and total outpatient costs were not significantly different regardless of whether patients switched treatments (Table 2). During the 6-month post-index period, mean total healthcare costs were significantly lower with apremilast versus biologics among patients who switched $(\$ 18,734$ vs. $\$ 29,995, P=0.0021)$ and did not switch treatments $(\$ 14,935$ vs. $\$ 21,665$,
Clinformatics ${ }^{\mathrm{TM}}$ Data Mart database. Standard prices are estimated. Allowed payment amounts (i.e., the insurance payment amounts) are across all provider services. USD US dollar

$P<0.0001)$; however, during the 18-month post-index period, mean total healthcare costs were significantly lower with apremilast only among patients who did not switch $(\$ 35,993$ vs. $\$ 50,867, P<0.0001$ ) (Fig. 4). At 6 months, mean total outpatient pharmacy costs were significantly lower for apremilast users versus biologic users regardless of treatment switching, whereas no significant differences in mean inpatient and total outpatient costs were observed (Table 2). At 18 months, mean total outpatient pharmacy costs were significantly lower for apremilast users versus biologic users only among patients who did not switch treatments; mean inpatient and total outpatient costs were not significantly different between treatments regardless of whether patients switched (Table 2). Within each treatment group, patients who switched treatments had higher healthcare costs at all time points compared with patients who did switch in all cost categories, with the exception of inpatient costs.

\section{PPPM Costs Among Patients Who Switched Treatments}

Mean PPPM healthcare costs were significantly lower regardless of treatment switching in the apremilast versus the biologic cohort during the 12-month post-index period (switch: $\$ 3315$ vs. $\$ 4291, P=0.0040$; no switch: $\$ 2164$ vs. $\$ 3141$, 


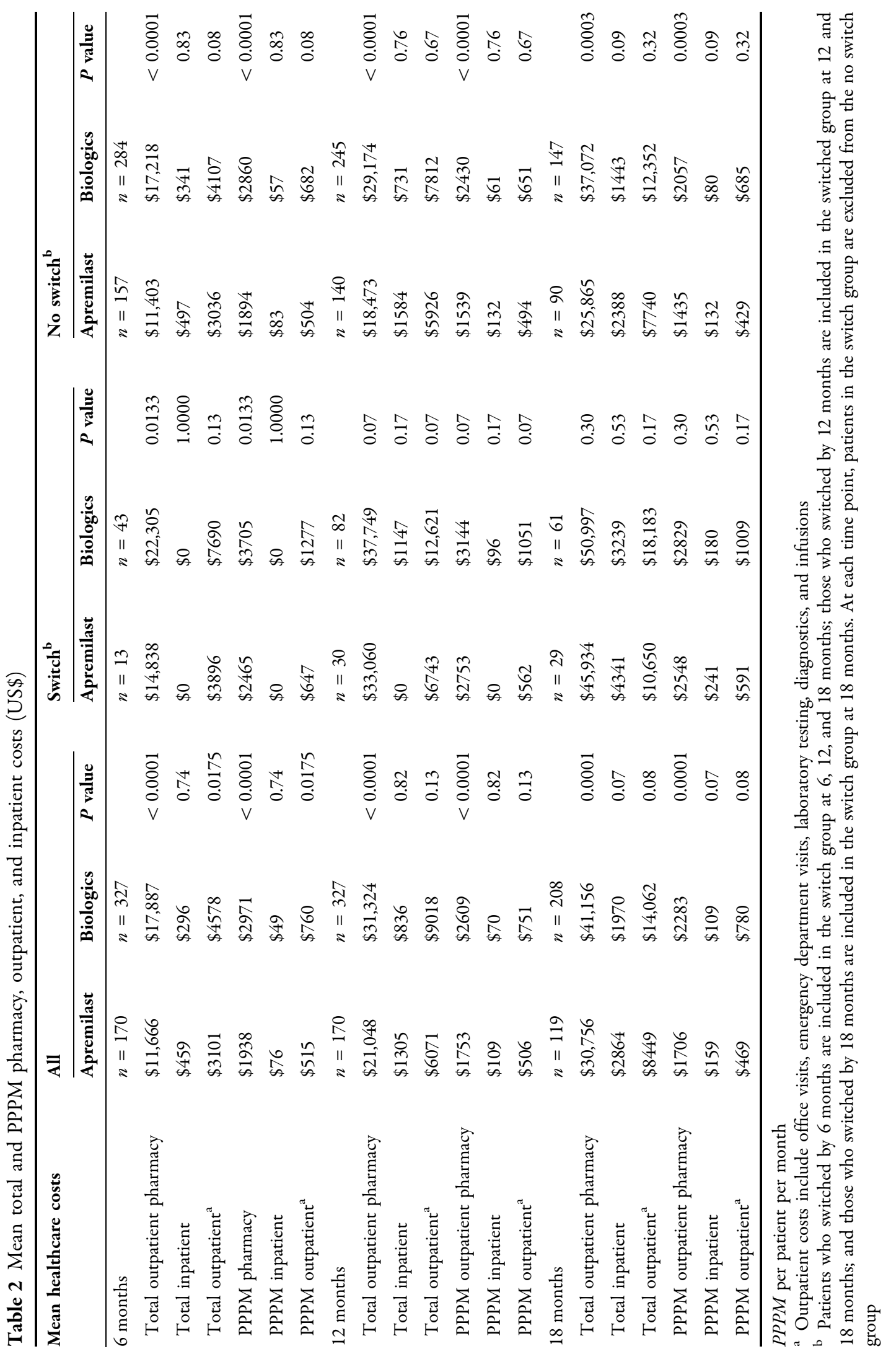




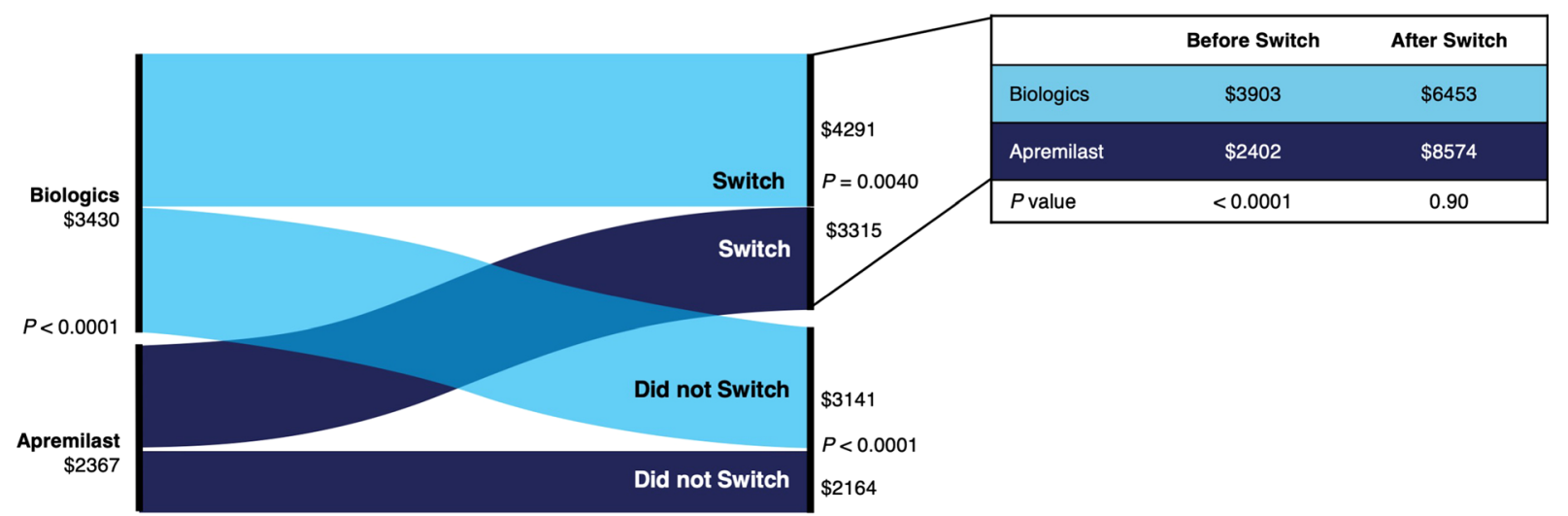

Fig. 5 Mean per patient per month healthcare $\operatorname{costs}^{a}$ overall and by treatment pattern over the 12 -month postindex period. ${ }^{a}$ Optum's standard pricing algorithms are applied to the de-identified claims data in the

$P<0.0001)$. Significantly lower mean PPPM outpatient pharmacy costs were observed with apremilast versus biologics only among patients who did not switch treatments, not among those who switched; no significant differences were seen in mean PPPM inpatient and mean PPPM outpatient costs (Table 2). During the 6-month post-index period, mean PPPM healthcare costs were significantly lower with apremilast versus biologics regardless of whether patients switched (\$3112 vs. \$4983, $P=0.0021)$ or did not switch (\$2841 vs. $\$ 3599$, $P<0.0001)$ treatments. During the 18-month post-index period, significantly lower mean PPPM healthcare costs were observed among apremilast users who did not switch treatments versus biologic users who did not switch treatments (\$1997 vs. \$2822, $P<0.0001$ ); among patients who switched treatments, mean PPPM healthcare costs were not significantly different ( $\$ 3380$ vs. $\$ 4017, P=0.19$ ). At 6 months, mean PPPM outpatient pharmacy costs were significantly different between treatments at 6 months regardless of whether patients switched treatments (Table 2). At 18 months, mean PPPM outpatient pharmacy costs were significantly lower with apremilast versus biologics only among patients who did not switch; no significant differences were seen in PPPM inpatient and outpatient costs regardless of a switch (Table 2).
Clinformatics ${ }^{\mathrm{TM}}$ Data Mart database. Standard prices are estimated. Allowed payment amounts (i.e., the insurance payment amounts) are across all provider services

Among patients who switched treatments, the PPPM healthcare cost before switch was lower for apremilast users versus biologic users (\$2402 vs. \$3903, $P<0.0001)$ during the 12-month post-index period (Fig. 5). Mean PPPM outpatient pharmacy costs were lower in the apremilast versus biologic cohort before the switch ( $\$ 2063$ vs. $\$ 3138, P<0.0001)$. Before switching, there were no mean PPPM inpatient costs in either cohort, and mean PPPM outpatient costs were significantly lower among apremilast users compared with biologic users (\$339 vs. $\$ 765, P=0.0159$ ). Mean PPPM healthcare costs were not significantly different in the apremilast and biologic cohorts after switching (Fig. 5), including mean PPPM outpatient pharmacy ( $\$ 7924$ vs. $\$ 5097, P=0.18$ ), PPPM inpatient ( $\$ 0$ vs. $\$ 132, P=0.17$ ), and PPPM outpatient costs $(\$ 650$ vs. $\$ 1224$, $P=0.24)$. Within each treatment group, patients who did not switch treatments had numerically lower PPPM healthcare costs compared with patients who switched at 6,12 , and 18 months in all categories except PPPM inpatient costs (Table 2).

\section{DISCUSSION}

This real-world, retrospective, administrative claims analysis compared treatment patterns 
and healthcare costs among biologic-naive patients with PsA who initiated treatment with apremilast or a biologic. Lack of treatment effectiveness and tolerability issues can affect whether patients remain on treatment, and treatment switch rates can serve as claims-based measures of treatment effectiveness and add to the understanding of patient treatment patterns. Our findings show similar treatment adherence and similar or lower switch rates for apremilast users compared with biologic users. Total and PPPM healthcare costs were significantly lower for apremilast users, even if they switched to a biologic during the 12-month post-index period, most notably because of differences in outpatient pharmacy costs. In the apremilast and biologic cohorts, patients who switched treatments had higher healthcare costs compared with those who did not switch from their index treatment.

Our findings are consistent with prior analyses among commercially insured, biologicnaive patients with psoriasis, which found similar treatment persistence rates and switch rates and significantly lower total healthcare costs at 12 months among patients initiating apremilast compared with those initiating a biologic $[32,33]$. To our knowledge, there are no similar studies in the literature conducted in patients with PsA who were treated with apremilast. Switch rates for patients in the biologic cohort were generally similar to those previously reported in the literature for biologic-treated patients with PsA [36]. These findings are in line with guidelines from the Group for Research and Assessment of Psoriasis and Psoriatic Arthritis (GRAPPA) and American College of Rheumatology, which recommend that patients who experience a lack of response or tolerability issues with biologic therapy should switch to another biologic or to a treatment with a different mechanism of action $[29,37,38]$. Mean adherence rates among patients in the apremilast and biologic cohorts in our study (PDC 0.84 and 0.82 , respectively) were higher than mean adherence rates in a recent claims-based analysis of patients with PsA receiving treatment with biologic agents (PDC ranging from 0.49 to 0.67) [39]. These differences may be due to variations in treatment history or other patient characteristics between the study populations.

Apremilast has favorable tolerability in clinical trials [13], and our results confirm this in a real-world setting, as evidenced by the observed treatment switch and adherence rates. Our results also provide a real-world benchmark for total costs of treatment with apremilast versus biologics among patients with PsA who did not switch during the 12 months post-index $(\$ 25,984$ vs. $\$ 37,717)$. These results are generally consistent with previously published estimates of the mean total yearly cost of apremilast treatment $(\$ 27,375)$ [40], and estimates of the 1-year treatment cost of biologics for patients with PsA alone (ranging from $\$ 26,916$ to $\$ 31,974$ ) or both psoriasis and PsA (ranging from $\$ 29,376$ to $\$ 34,541$ ) [41].

Over 12 months post-index, we observed lower mean PPPM healthcare costs in the apremilast cohort compared with the biologic cohort (\$2367 vs. \$3430). Considering that PPPM healthcare costs were significantly lower with apremilast versus biologics at 12 months, the lower drug acquisition cost for apremilast likely contributed to the reduced annual healthcare costs. We also observed that among patients who switched treatments, those who switched from apremilast had lower healthcare costs before switching compared with patients who switched from a biologic, whereas healthcare costs after switching were similar in the two cohorts. This finding is consistent with the fact that patients switching from apremilast were switching to a more costly biologic agent, whereas patients switching from a biologic were not necessarily switching to a less costly treatment. Palmer et al. [42] demonstrated that patients with PsA who switched from a first-line anti-TNF agent had higher PPPM pharmacy costs with each successive treatment switch compared with patients who did not switch. Taken together, these findings suggest that starting treatment with apremilast may be an effective and cost-effective strategy for managing PsA in biologic-naive patients, regardless of whether they later switch to a biologic agent. These findings add to the current knowledge of health economic outcomes with apremilast treatment in patients with PsA [43] and may 
help providers and payers improve patient outcomes and reduce healthcare costs.

\section{Limitations}

This study was limited to individuals with United Healthcare commercial health coverage or Medicare Advantage, and our findings may not be generalizable to patients with PsA with other insurance plans or without health insurance coverage. Patients with an apremilast claim were included regardless of dosage strength or schedule, and some patients may have received apremilast or a biologic with a dosing regimen that differs from the approved dose/schedule in the package insert. The Optum CDM database relies on administrative claims data, which are subject to coding limitations and entry error. In addition, administrative claims data do not provide clinical details such as disease severity or activity, and unmeasured baseline disease severity or activity may contribute to observed differences in costs. While propensity score matching controlled for differences between the two exposure cohorts, adjustment was limited to characteristics that could be measured from administrative claims. In the comparison of treatment switchers versus non-switchers, patient characteristics may not have been well balanced given that the propensity scores did not seek balance on switching status. Furthermore, medication adherence measures were based on filled prescriptions, and it was not possible to confirm whether patients actually took the medication as directed. This analysis did not assess reasons for switching and was limited to claims-based definitions of a switch. The possibility of underdiagnosis of PsA may lead to selection bias and/or smaller sample sizes, as patients with PsA who were untreated or did not have a relevant diagnosis recorded on their medical claims were excluded. It also is possible that apremilast is prescribed to patients without markers of severe disease, whereas biologic agents may be prescribed for a broad range of patients with active PsA [12]. Patients who have more severe disease tend to utilize more healthcare resources and also have higher healthcare costs [11]. Because claims for PsA do not provide details on disease severity, our findings may not be generalizable across populations of patients with different levels of PsA clinical disease severity. However, because our baseline costs and patient characteristics were well balanced between the groups post-switch, this concern may be lessened. Findings of treatment patterns and costs may not be generalizable to those patients with previous biologic exposure. This study did not evaluate whether differences in healthcare costs between patients initiating apremilast versus a biologic may emerge after the 18-month duration of follow-up. Assessments of effectiveness between apremilast and biologics were limited to treatment patterns and costs. Furthermore, not all biologic agents currently available for the treatment of PsA were included because some were not approved by the FDA at the time of the study. Additionally, newer biologic agents that were included in the analysis had small sample sizes. As a result of varying and shifting utilization management criteria (e.g., fewer prior authorizations), additional studies should be performed to assess the effectiveness of newer agents and subgroups of biologics (i.e., TNF $\alpha$ or IL inhibitors), consistency of results across multiple settings, and changes in treatment patterns over time. This study did not assess the impact of comorbidities on study outcomes. Also, the presence of comorbidities can be a confounding variable; however, we controlled for between-group differences in comorbidities through propensity score matching, which included the Charlson comorbidity index score as a variable.

\section{CONCLUSION}

In this real-world analysis, biologic-naive patients with PsA who initiated treatment with apremilast had similar or lower switch rates and similar adherence rates versus those who initiated treatment with a biologic; furthermore, PPPM healthcare costs were significantly lower in the apremilast cohort, regardless of whether patients switched therapies during the post-index period. The cost advantage demonstrated with apremilast treatment was driven by lower 
pharmacy costs. These findings provide additional context on the effectiveness and cost-effectiveness of apremilast relative to biologic agents in biologic-naive patients with PsA and may help physicians and payers better evaluate the positioning of apremilast among other available therapies for PsA. Future studies with larger sample sizes are needed to increase our understanding of switch rates and to identify potential predictors of switching from one agent to another. Because comorbidities can influence initial treatment choices and treatment switching among patients with PsA [37], further studies on the impact of comorbidities on switch rates with apremilast and biologic agents are warranted.

\section{ACKNOWLEDGEMENTS}

Funding. This study was sponsored by Celgene Corporation, Summit, NJ, USA. The study sponsor also funded the Rapid Service and Open Access Fees. All authors had full access to all of the data in this study and take complete responsibility for the integrity of the data and accuracy of the data analysis.

Editorial Assistance. Editorial assistance in the preparation of this manuscript was provided by Larry Radican, Ph.D., MPH, of Peloton Advantage, LLC, an OPEN Health company, Parsippany, NJ, USA. Support for this assistance was funded by Celgene Corporation. The authors, however, directed and are fully responsible for all content and editorial decisions for this manuscript.

Authorship. All named authors meet the International Committee of Medical Journal Editors (ICMJE) criteria for authorship for this article, take responsibility for the integrity of the work as a whole, and have given their approval for this version to be published.

Disclosures. Jashin J. Wu has served an investigator for AbbVie, Amgen, Eli Lilly, Janssen, and Novartis; a consultant for AbbVie, Almirall, Amgen, Bristol-Myers Squibb, Celgene
Corporation, Dermira, Dr. Reddy's Laboratories, Eli Lilly, Janssen, LEO Pharma, Novartis, Promius Pharma, Regeneron, Sun, UCB, and Valeant; and a speaker for AbbVie, Celgene Corporation, Novartis, Sun, UCB, and Valeant. Corey Pelletier, Brian Ung, and Marc Tian are employees of Bristol-Myers Squibb and were employees of Celgene Corporation at the time of study conduct. Ibrahim Khilfeh is an employee of Amgen Inc. and was an employee of Celgene Corporation at the time of study conduct. Jeffrey R. Curtis has received grant/ research support from AbbVie, Amgen, Eli Lilly, Janssen, and Novartis and has served as a speaker for AbbVie, Almirall, Amgen, BMS, Celgene Corporation, Dermira, Dr. Reddy's Laboratories, Eli Lilly, Janssen, LEO Pharma, Novartis, Ortho Dermatologics, Promius Pharma, Regeneron, Sun, UCB, and Valeant.

Compliance with Ethics Guidelines. The CDM database provides detailed utilization, outcomes, and cost data for healthcare services provided in the inpatient, outpatient, and outpatient pharmacy settings and is statistically deidentified in accordance with the Health Insurance Portability and Accountability Act of 1996 as per 45 Code of Federal Regulations 164.506(d) (2)(ii)(B). Institutional review board approval was not required because this study used only de-identified patient records and did not involve the collection, use, or transmittal of individually identifiable data, as dictated by Title 45 Code of Federal Regulations 46.101(b)(4).

Data Availability. The datasets generated during and/or analyzed during the current study are not publicly available as the study was conducted in a database that requires a license to access but are available from the corresponding author on reasonable request.

Open Access. This article is licensed under a Creative Commons Attribution-NonCommercial 4.0 International License, which permits any non-commercial use, sharing, adaptation, distribution and reproduction in any medium or format, as long as you give appropriate credit to the original author(s) and the source, provide 
a link to the Creative Commons licence, and indicate if changes were made. The images or other third party material in this article are included in the article's Creative Commons licence, unless indicated otherwise in a credit line to the material. If material is not included in the article's Creative Commons licence and your intended use is not permitted by statutory regulation or exceeds the permitted use, you will need to obtain permission directly from the copyright holder. To view a copy of this licence, visit http://creativecommons.org/licenses/by$\mathrm{nc} / 4.0 /$.

\section{REFERENCES}

1. Gladman DD, Antoni C, Mease P, Clegg DO, Nash P. Psoriatic arthritis: epidemiology, clinical features, course, and outcome. Ann Rheum Dis. 2005;64(Suppl 2):ii14-7.

2. Sakkas LI, Alexiou I, Simopoulou T, Vlychou M. Enthesitis in psoriatic arthritis. Semin Arthritis Rheum. 2013;43:325-34.

3. Ritchlin CT, Colbert RA, Gladman DD. Psoriatic arthritis. N Engl J Med. 2017;376:957-70.

4. McDonough E, Ayearst R, Eder L, et al. Depression and anxiety in psoriatic disease: prevalence and associated factors. J Rheumatol. 2014;41:887-96.

5. Mease PJ, Armstrong AW. Managing patients with psoriatic disease: the diagnosis and pharmacologic treatment of psoriatic arthritis in patients with psoriasis. Drugs. 2014;74:423-41.

6. Walsh JA, McFadden ML, Morgan MD, et al. Work productivity loss and fatigue in psoriatic arthritis. J Rheumatol. 2014;41:1670-4.

7. Ogdie A, Schwartzman S, Husni ME. Recognizing and managing comorbidities in psoriatic arthritis. Curr Opin Rheumatol. 2015;27:118-26.

8. Feldman SR, Tian H, Gilloteau I, Mollon P, Shu M. Economic burden of comorbidities in psoriasis patients in the United States: results from a retrospective U.S. database. BMC Health Serv Res. 2017;17:337.

9. Merola JF, Herrera V, Palmer JB. Direct healthcare costs and comorbidity burden among patients with psoriatic arthritis in the USA. Clin Rheumatol. 2018;37:2751-61.
10. D'Angiolella LS, Cortesi PA, Lafranconi A, et al. Cost and cost effectiveness of treatments for psoriatic arthritis: a systematic literature review. Pharmacoeconomics. 2018;36:567-89.

11. Burgos-Pol R, Martinez-Sesmero JM, Ventura-Cerda JM, Elias I, Caloto MT, Casado MA. The cost of psoriasis and psoriatic arthritis in 5 European countries: a systematic review. Actas Dermosifiliogr. 2016;107:577-90.

12. Gossec L, Smolen JS, Ramiro S, et al. European League Against Rheumatism (EULAR) recommendations for the management of psoriatic arthritis with pharmacological therapies: 2015 update. Ann Rheum Dis. 2016;75:499-510.

13. Kavanaugh A, Mease PJ, Gomez-Reino JJ, et al. Treatment of psoriatic arthritis in a phase 3 randomized, placebo-controlled trial with apremilast, an oral phosphodiesterase 4 inhibitor. Ann Rheum Dis. 2014;73:1020-6.

14. Stelara (ustekinumab) [prescribing information]. Horsham: Janssen Biotech, Inc; 2018. janssenlabels. com/package-insert/product-monograph/prescribinginformation/STELARA-pi.pdf.

15. Cosentyx (secukinumab) [package insert]. East Hanover: Novartis Pharmaceuticals Corporation; 2016. pharma.us.novartis.com/sites/www.pharma. us.novartis.com/files/cosentyx.pdf.

16. Taltz (ixekizumab) [package insert]. Indianapolis: Eli Lilly and Company; 2017. uspl.lilly.com/taltz/ taltz.html\#pi.

17. Singh JA, Guyatt G, Ogdie A, et al. 2018 American College of Rheumatology/National Psoriasis Foundation guideline for the treatment of psoriatic arthritis. Arthritis Rheumatol. 2019;71:5-32.

18. Menter A, Strober BE, Kaplan DH, et al. Joint AADNPF guidelines of care for the management and treatment of psoriasis with biologics. J Am Acad Dermatol. 2019;80:1029-72.

19. Menter A, Korman NJ, Elmets CA, et al. Guidelines of care for the management of psoriasis and psoriatic arthritis: section 6 . Guidelines of care for the treatment of psoriasis and psoriatic arthritis: casebased presentations and evidence-based conclusions. J Am Acad Dermatol. 2011;65:137-74.

20. Otezla (apremilast) [package insert]. Summit: Celgene Corporation; 2019. media2.celgene.com/ content/uploads/otezla-pi.pdf.

21. Schafer P. Apremilast mechanism of action and application to psoriasis and psoriatic arthritis. Biochem Pharmacol. 2012;83:1583-90. 
22. Zerilli T, Ocheretyaner E. Apremilast (Otezla): a new oral treatment for adults with psoriasis and psoriatic arthritis. P T. 2015;40:495-500.

23. Coates LC, FitzGerald O, Helliwell PS, Paul C. Psoriasis, psoriatic arthritis, and rheumatoid arthritis: is all inflammation the same? Semin Arthritis Rheum. 2016;46:291-304.

24. Cutolo M, Myerson GE, Fleischmann R, et al. A phase III, randomized, controlled trial of apremilast in patients with psoriatic arthritis: results of the PALACE 2 trial. J Rheumatol. 2016;43:1724-34.

25. Edwards CJ, Blanco FJ, Crowley J, et al. Apremilast, an oral phosphodiesterase 4 inhibitor, in patients with psoriatic arthritis and current skin involvement: a phase III, randomised, controlled trial (PALACE 3). Ann Rheum Dis. 2016;75:1065-73.

26. Paul C, Cather J, Gooderham M, et al. Efficacy and safety of apremilast, an oral phosphodiesterase 4 inhibitor, in patients with moderate to severe plaque psoriasis over 52 weeks: a phase III, randomized, controlled trial (ESTEEM 2). Br J Dermatol. 2015;173:1387-99.

27. Feldman SR, Goffe B, Rice G, et al. The challenge of managing psoriasis: unmet medical needs and stakeholder perspectives. Am Health Drug Benefits. 2016;9:504-13.

28. Lebwohl MG, Bachelez H, Barker J, et al. Patient perspectives in the management of psoriasis: results from the population-based Multinational Assessment of Psoriasis and Psoriatic Arthritis survey. J Am Acad Dermatol. 2014;70:871-81.

29. Merola JF, Lockshin B, Mody EA. Switching biologics in the treatment of psoriatic arthritis. Semin Arthritis Rheum. 2017;47:29-37.

30. Glintborg B, Ostergaard M, Dreyer L, et al. Treatment response, drug survival, and predictors thereof in 764 patients with psoriatic arthritis treated with anti-tumor necrosis factor alpha therapy: results from the nationwide Danish DANBIO registry. Arthritis Rheum. 2011;63:382-90.

31. Mayba JN, Gooderham MJ. Real-world experience with apremilast in treating psoriasis. J Cutan Med Surg. 2017;21:145-51.

32. Feldman SR, Pelletier CL, Wilson KL, et al. Realworld US healthcare costs of psoriasis for biologicnaive patients initiating apremilast or biologics. J Comp Eff Res. 2019;8:45-54.

33. Wu JJ, Pelletier C, Ung B, Tian M. Real-world treatment patterns and healthcare costs among biologic-naive patients initiating apremilast or biologics for the treatment of psoriasis. J Med Econ. 2019;22:365-71.

34. Austin PC. A comparison of 12 algorithms for matching on the propensity score. Stat Med. 2014;33:1057-69.

35. Feldman SR, Tian H, Wang X, Germino R. Health care utilization and cost associated with biologic treatment patterns among patients with moderate to severe psoriasis: analyses from a large U.S. claims database. J Manag Care Spec Pharm. 2019;25: 479-88.

36. Costa L, Perricone C, Chimenti MS, et al. Switching between biological treatments in psoriatic arthritis: a review of the evidence. Drugs R\&D. 2017;17: 509-22.

37. Coates LC, Kavanaugh A, Mease PJ, et al. Group for Research and Assessment of Psoriasis and Psoriatic Arthritis: treatment recommendations for psoriatic arthritis 2015. Arthritis Rheumatol. 2016;68: 1060-71.

38. Singh JA, Guyatt G, Ogdie A, et al. Special article: 2018 American College of Rheumatology/National Psoriasis Foundation guideline for the treatment of psoriatic arthritis. Arthritis Care Res. 2019;71:2-29.

39. Oelke KR, Chambenoit O, Majjhoo AQ, Gray S, Higgins K, Hur P. Persistence and adherence of biologics in US patients with psoriatic arthritis: analyses from a claims database. J Comp Eff Res. 2019;8:607-21.

40. Cheng J, Feldman SR. The cost of biologics for psoriasis is increasing. Drugs Context. 2014;3: 212266.

41. Chastek B, White J, Van Voorhis D, Tang D, Stolshek BS. A retrospective cohort study comparing utilization and costs of biologic therapies and JAK inhibitor therapy across four common inflammatory indications in adult US managed care patients. Adv Ther. 2016;33:626-42.

42. Palmer JB, Li Y, Herrera V, Liao M, Tran M, Ozturk ZE. Treatment patterns and costs for anti-TNFalpha biologic therapy in patients with psoriatic arthritis. BMC Musculoskelet Disord. 2016;17:261.

43. Betts KA, Griffith J, Friedman A, Zhou ZY, Signorovitch JE, Ganguli A. An indirect comparison and cost per responder analysis of adalimumab, methotrexate and apremilast in the treatment of methotrexate-naive patients with psoriatic arthritis. Curr Med Res Opin. 2016;32:721-9. 